\section{Assistência à criança: preenchimento da caderneta de saúde em municípios do semi- árido brasileiro}

\section{Child healthcare: completion of health records in municipalities in the semi-arid region of Brazil}

Juvenal Soares Dias da Costa 1

Juraci Almeida Cesar 2

Marcos Paschoal Pattussi 3

Larissa Prado da Fontoura 4

Lidiane Barazzetti 5

Marcelo Felipe Nunes 6

Mari Ângela Gaedke 7

Rosângela Uebel 8

13-8 Programa de Pós-Graduação em Saúde Coletiva. Universidade do Vale do Rio dos Sinos. Av. Unisinos, 950. Caixa Postal 275. São Leopoldo, RS, Brasil. CEP: 93.022-000.

E-mail: episoares@terra.com.br

2 Divisão de População \& Saúde. Universidade Federal do Rio Grande. Rio Grande, RS, Brasil.

\section{Resumo}

Objetivos: determinar a proporção de crianças

Objectives: to determine the proportion of children with adequately completed health records and to investigate associations with geographical, socioeconomic and the mother and child's biological characteristics, and with the percentage use of health services in two municipalities in the semi-arid region of Brazil.

Methods: a population-based cross-sectional study was carried with children aged up to five years. Data were collected by way of a household questionnaire between July and September 2008. The presence of all records relating to identification, growth, development and vaccine status of the child was deemed to be adequate completion. The raw and adjusted prevalence ratios were calculated using the Poisson Regression, following a hierarchized model.

Results: of the 342 children studied, $22.2 \%$ (CI95\% 17.5 - 26.2) had adequately completed health records. A high level of education of the mother was associated with this, after adjusting for the effect of variables in the hierarchized model.

Conclusions: the results show a low level of adequate completion of health records. The high prevalence of inadequately completed records in mothers with low levels of education may reflect inequalities in the local health system. Children have a right to adequate completion of health records and failure to do so suggests lack of training of health workers and parents in basic health care needs.

Key words Primary health care, Child health, Health equity, Child development com cadernetas de saúde com preenchimento adequado e analisar associações com características geográficas, socioeconômicas e biológicas maternas e da criança e com o percentual de utilização de serviços de saúde em dois municípios do semi-árido brasileiro.

Métodos: estudo transversal de base populacional com crianças até cinco anos de idade. A coleta de dados foi realizada mediante aplicação de questionários domiciliar entre julho e setembro de 2008. A presença de todos os registros na caderneta, relativos à identificação, crescimento, desenvolvimento e status vacinal da criança foi definida como preenchimento adequado. Calcularam-se as razões de prevalência brutas e ajustadas pela Regressão de Poisson, seguindo modelo hierarquizado.

Resultados: entre as 342 crianças estudadas, 22,2\% (IC95\% 17,5 a 26,2) apresentavam cadernetas de saude preenchidas de forma adequada. A alta escolaridade materna esteve associada a este evento, após ajuste do efeito das variáveis no modelo hierarquizado.

Conclusões: os resultados demonstraram baixo percentual de cadernetas de saúde adequadamente preenchidas. A maior prevalência de cadernetas com preenchimento inadequado nas mães de baixa escolaridade pode denotar iniquidade no sistema de saúde local. O preenchimento adequado da caderneta um direito da criança, a sua inadequação indicou despreparo dos profissionais de saúde e dos pais às necessidades da atenção básica.

Palavras-chave Atenção primária a saúde, Saúde da criança, Equidade em saúde, Desenvolvimento infantil 


\section{Introdução}

A redução da mortalidade infantil é um dos principais objetivos das políticas de saúde para a infância nos países em desenvolvimento, sendo a vigilância da mortalidade infantil e fetal e o acompanhamento do crescimento infantil um dos Objetivos de Desenvolvimento do Milênio propostos pela Organização das Nações Unidas (ONU) que devem ser atingidos por todos os países até 2015.1,2

$\mathrm{Na}$ tentativa de diminuir a mortalidade infantil, desde 1984 o Brasil vem promovendo políticas de atenção integral à saúde das crianças, promovendo acompanhamento do crescimento, do desenvolvimento, incentivo ao aleitamento materno e das imunizações, tentando padronizar o cuidado nas doenças respiratórias e diarréicas. 3 Entretanto, a diminuição da mortalidade infantil no Brasil está relacionada às melhorias nas condições de vida e na atenção à saúde da criança. 1 Porém esses avanços não têm ocorrido igualmente em todos os Estados brasileiros,observando-se declínio menos acentuado em locais mais vulneráveis, como a Região Nordeste, particularmente a região do semi-árido, onde as taxas de mortalidade infantil podem atingir o dobro das encontradas nas Regiões Sul, Sudeste e Centro-Oeste. ${ }^{4}$

Assim, ações de saúde voltadas à redução da mortalidade infantil nestas regiões se tornam prioritárias, entre elas o correto preenchimento e uso da caderneta de saúde da criança. 5

A caderneta de saúde da criança é um instrumento importante para o acompanhamento da criança e o seu correto preenchimento é essencial para as ações de vigilância e de promoção da saúde infantil, para os pais e para as equipes de saúde. ${ }^{6} \mathrm{~A}$ caderneta permite a identificação precoce de problemas de saúde, como atraso de crescimento, desnutrição e obesidade, possibilitando a sua reversão antes que que evoluam para quadros crônicos ou irreversíveis. Trata-se, portanto, de um instrumento eficaz, de baixo custo de acompanhamento da saúde das crianças. ${ }^{6}$ Após revisão feita pelo Ministério da Saúde em 2005, o Cartão da Criança passou a se denominar Caderneta de Saúde da Criança, tendo sido incluídas orientações sobre alimentação saudável, gráficos de evolução do perímetro cefálico, além de orientações sobre a saúde auditiva, visual e bucal, prevenção de acidentes, espaço para anotações de intercorrências clínicas, tratamentos efetuados e da suplementação profilática de ferro e de vitamina A. Houve igualmente a extensão do período de acompanhamento da criança até os dez anos de idade, garantindo assim o cuidado integral até esta faixa etária.?

Estudos realizados em diferentes localidades no Brasil têm avaliado a qualidade da assistência materno-infantil mediante a verificação do grau de preenchimento das cadernetas de saúde das crianças. ${ }^{8,9}$ Pesquisa desenvolvida no interior do Estado de Minas Gerais, contatou que apenas 31\% das crianças estudadas possuíam cadernetas com registros de peso/idade nos gráficos de crescimento, não havendo registros do desenvolvimento neuropsicomotor em todas elas, sugerindo fragilidade da assistência. ${ }^{8}$ Em outro estudo conduzido na cidade de Pelotas (RS), mais de $90 \%$ das cadernetas possuíam gráficos de peso/idade preenchidos, porém constatou-se que apenas as informações relativas à identificação, crescimento e imunizações foram foco de atenção por parte dos profissionais de saúde. 9 Esses dados mostram a precariedade da assistência à saúde nessas localidade e mostram a necessidade de sensibilizar os profissionais de saúde para a importância da caderneta de saúde para o acompanhamento e promoção da saúde das crianças.

Este estudo teve por objetivos verificar a qualidade da assistência à saúde mediante a investigação da frequência de cadernetas de saúde da criança com preenchimento adequado e verificar a sua associação com possíveis variáveis explanatórias em duas cidades do semi-árido nordestino.

\section{Métodos}

Este artigo foi recorte de um estudo transversal de base populacional mais amplo, realizado pela Universidade Federal do Rio Grande (FURG), que teve por objetivo avaliar indicadores básicos de saúde materno-infantil nos municípios de Caracol e Anísio de Abreu, no Piauí. ${ }^{10,11}$ A coleta de dados foi realizada entre os meses de julho a setembro de 2008. A população alvo foi constituída por todas as crianças com idades até cinco anos de idade residentes nas áreas urbana e rural destes municípios.

Os municípios supracitados estão localizados no sul do Estado do Piauí, a cerca de $600 \mathrm{~km}$ da capital Teresina. Conforme estimativas do IBGE em 2010, a população de Caracol e de Anísio de Abreu era de 10.212 e 9.098 habitantes, respectivamente. O Índice de Desenvolvimento Humano (IDH) do município de Caracol era de 0,59; o Produto Interno Bruto (PIB) per capita de $\mathrm{R} \$ 2.618,38^{2}$; a taxa de mortalidade infantil de 26,3/1000 nascidos vivos. $12 \mathrm{O}$ município contava com cinco estabelecimentos de saúde, sendo quatro públicos e um privado. Em Anísio de Abreu, o IDH era de 0,63; o PIB per capita de R $\$ 2.469,67(2008)^{2}$ e a taxa de mortali- 
dade infantil de 18,0/1000.12 O município contava com quatro estabelecimentos de saúde, todos públicos. O IDH dos municípios pesquisados figurava entre os piores do país. Em 2008, cerca da metade da população vivia abaixo da linha da pobreza. ${ }^{2}$

Em ambos os municípios, as quadras nas áreas urbanas foram numeradas, e nas áreas rurais, os limites foram delimitados por estradas, riachos e montanhas para facilitar a logística e distribuir os domicílios entre os entrevistadores. Em Caracol foram visitados todos os 2169 domicílios existentes, assim como os 1740 domicílios de Anísio de Abreu, tendo sido incluídas no estudo todas as crianças até cinco anos ali residentes. Foi excluída uma criança cuja mãe que apresentava limitações cognitivas e/ou físicas que impediu a aplicação do questionário.

Questionários padronizados e pré-codificados foram aplicados às mães ou responsáveis pela guarda das crianças, por dez entrevistadores treinados para a aplicação do questionário. Foi realizado controle de qualidade durante o período de coleta de dados por meio de repetição parcial de $5 \%$ das entrevistas ou supervisão direta.

O número de crianças estudadas foi suficiente para se estimar um percentual de preenchimento adequado das cadernetas da criança de $22 \%$, admitindo-se erro aceitável de $4 \%$ e um nível de confiança de $95 \%$. Para se testar associações com nível de confiança de $95 \%$, poder de $80 \%$, razão de não expostos:expostos de 1:9 (correspondente a distribuição de renda), prevalência do desfecho no grupo não exposto de $30 \%$ e com risco de 2,0 seriam necessárias 263 crianças.

O desfecho do estudo foi a "adequação do preenchimento da caderneta de saúde da criança", que foi definido pelo preenchimento conjunto das informações relativas à identificação, crescimento (peso e altura), desenvolvimento e imunização.

As variáveis independentes foram classificadas como: geográficas (município de residência; área urbana ou rural; distância da residência ao serviço de saúde); características biológicas maternas (cor da pele referida pela mãe; idade e número de filhos tidos); características socioeconômicas da família (renda familiar, escolaridade materna; escolaridade paterna e beneficiária do Programa Bolsa Família); características biológicas da criança (idade e peso ao nascimento); utilização de serviços de saúde (realização do pré-natal; pré-natal adequado; consulta puerperal; consulta da criança nos últimos três meses, consulta de puericultura nos últimos três meses e hospitalização no último ano). O pré-natal foi classificado como adequado quando iniciado no primeiro trimestre de gestação e com a realização de seis ou mais consultas. ${ }^{13}$

A entrada dos dados foi realizada através do Programa Epi Info versão 6.04, com dupla entrada, para posterior comparação dos bancos de dados, correção dos possíveis erros de digitação e limpeza. Durante o processamento dos dados, foram checadas consistência e validade interna dos dados pelos pesquisadores responsáveis pelo estudo.

As análises foram realizadas utilizando os Programas SPSS versão 17 e STATA versão 11.0. A associação entre o correto preenchimento da caderneta e os fatores investigados foi testada por meio do teste do qui-quadrado de Pearson e exato de Fisher, com nível de significância $<0,05$. A análise bruta e ajustada foi realizada por meio de Regressão de Poisson, 14 considerando a frequência elevada do desfecho do evento. A medida de efeito foi obtida pelo cálculo da razão de prevalência.

A análise de regressão múltipla foi realizada aplicando um modelo hierarquizado. 15 No $1^{\circ}$ nível, mais distal em relação ao desfecho, foram incluídas as variáveis geográficas, características biológicas da mãe e características socioeconômicas da família. O $2^{\circ}$ nível foi constituído pelas variáveis de características biológicas da criança. No $3^{\circ}$ nível, mais proximal em relação ao desfecho, incluíram-se as variáveis em relação à utilização de serviços de saúde. Todos os níveis determinavam os níveis inferiores e tinham efeito direto sobre o desfecho. Para controle de fatores de confusão, foram levadas para a análise ajustada as variáveis que na análise bruta tiveram $p<0,20$, e mantidas no modelo apenas as variáveis com valor $p<0,05$.

O projeto de pesquisa foi submetido e aprovado pelo Comitê de Ética em Pesquisa da participantes assinaram o Termo de Consentimento Livre e Esclarecido (TCLE).

\section{Resultados}

Das 1640 crianças localizadas, 1551 (94,6\%) apresentaram algum tipo de caderneta da criança (cartão da criança, cartão de vacinas). Foram excluídas da análise 1209 crianças que apresentavam outro modelo de caderneta que não apresentava seções para o acompanhamento do desenvolvimento e 89 crianças que não possuíam caderneta. Desta forma, foram analisadas 342 crianças.

Entre as 342 cadernetas, $327(95,6 \%)$ apresentavam dados de identificação, 104 (30,4\%) observações de acompanhamento do desenvolvimento, $242(70,8 \%)$ algum peso anotado e $314(91,8 \%)$ tinham registro de imunizações. Desta forma, 76 
(22,2\%; IC95\% 17,5 a 26,2) cadernetas estavam adequadamente preenchidas.

Quanto às variáveis geográficas, verificou-se predomínio das crianças residindo no município de Caracol $(55,3 \%) ; 53,2 \%$ vivendo na área rural e $62,1 \%$ morando até três $\mathrm{km}$ de distância de algum serviço de saúde. Em relação às variáveis socioeconômicas, a maioria das famílias tinha renda menor que um salário mínimo $(65,5 \%)$, a escolaridade materna situava-se entre cinco a oito $(41,2 \%)$ e a escolaridade paterna era de até quatro anos $(48,5 \%)$ e $33,6 \%$ das famílias eram beneficiárias do Programa Bolsa Família. A análise bruta das variáveis geográficas mostrou maior prevalência de adequação de preenchimento da caderneta nos moradores da zona urbana. Quanto às variáveis socioeconômicas, as maiores prevalências de preenchimento adequado foram observadas nas crianças com mães e pais com escolaridade mais elevada. Observou-se prevalência $63 \%$ maior de adequação no preenchimento da caderneta naquelas crianças que não estavam inscritas no programa Bolsa Família (Tabela 1).

Em relação às características biológicas maternas, houve predomínio da faixa etária entre 20 e 29 anos $(57,3 \%)$, cor da pele parda e mulata $(83,9 \%)$ e mães com apenas um filho $(43,9 \%)$.

Quanto às características biológicas das crianças, $56,2 \%$ tinham menos de um ano de idade e 4,7\% tinham apresentado baixo peso ao nascer. As características biológicas maternas e das crianças não apresentaram associação estatisticamente significativa com o desfecho (Tabela 2). O resultado da análise bruta da variável peso ao nascimento não foi incluído na tabela, pois todas as crianças que apresentaram baixo peso ao nascer tinham caderneta de saúde preenchida como inadequada.

Tabela 1

Razão de prevalência bruta da associação do preenchimento adequado das cadernetas de saúde e características geográficas e socioeconômicas. Caracol e Anísio de Abreu, Piauí, 2008.

\begin{tabular}{|c|c|c|c|c|c|c|}
\hline Variáveis & $\mathbf{N}$ & $\%$ & $\begin{array}{l}\text { Prevalência de } \\
\text { adequação (\%) }\end{array}$ & $\mathbf{R P}$ & $\mathrm{IC} 95 \%$ & $p$ \\
\hline Município & & & & & & $0,12 *$ \\
\hline Caracol & 189 & 55,3 & 19,0 & 1,00 & $0,92-2,04$ & \\
\hline Anísio de Abreu & 153 & 44,7 & 26,1 & 1,37 & & \\
\hline Área & & & & & & $0,04 *$ \\
\hline Rural & 182 & 53,2 & 17,6 & 1,00 & $1,05-2,34$ & \\
\hline Urbana & 160 & 46,8 & 27,5 & 1,56 & & \\
\hline Distância do serviço de saúde $(\mathrm{km})$ & & & & & & 0,50 * \\
\hline Mais de 3 & 128 & 37,9 & 19,5 & 1,00 & $0,76-1,80$ & \\
\hline Até 3 & 210 & 62,1 & 22,9 & 1,17 & & \\
\hline Renda familiar (SM) & & & & & & $0,05 * *$ \\
\hline Menos de 1 & 224 & 65,5 & 19,2 & 1,00 & & \\
\hline 1 a 1,9 & 73 & 21,3 & 26,0 & 1,36 & $0,85-2,17$ & \\
\hline 2 ou mais & 45 & 13,2 & 31,1 & 1,62 & $0,97-2,70$ & \\
\hline Escolaridade da mãe (anos) & & & & & & $<0,001$ ** \\
\hline 0 a 4 & 9 & 26,9 & 7,6 & 1,00 & & \\
\hline 5 a 8 & 141 & 41,2 & 20,6 & 2,70 & $1,24-5,91$ & \\
\hline 9 ou mais & 109 & 31,9 & 36,7 & 4,82 & $2,27-10,25$ & \\
\hline Escolaridade do pai (anos) & & & & & & $0,01 * *$ \\
\hline 0 a 4 & 164 & 48,5 & 16,5 & 1,00 & & \\
\hline 5 a 8 & 85 & 25,1 & 21,2 & 1,29 & $0,75-2,20$ & \\
\hline 9 ou mais & 89 & 26,3 & 31,5 & 1,91 & $1,20-3,03$ & \\
\hline Bolsa Família & & & & & & $0,004 *$ \\
\hline Sim & 115 & 33,6 & 15,7 & 1,00 & $1,01-2,64$ & \\
\hline Não & 227 & 66,4 & 25,6 & 1,63 & & \\
\hline
\end{tabular}

* qui-quadrado $p$-valor de Pearson; ** qui-quadrado $p$-valor de linearidade. 
Quanto à utilização de serviços de saúde, verificou-se que $98,0 \%$ das mães realizaram pré-natal, das quais $80,0 \%$ foram classificados como inadequados. Um percentual de $71,6 \%$ das mulheres foi consultado no puerpério, enquanto que $58,2 \%$ das crianças foram consultadas nos últimos três meses, sendo que $2,6 \%$ foram consultas de puericultura. Entre as crianças, 8,5\% haviam sido hospitalizadas nos últimos 12 meses. A análise das variáveis relacionadas à utilização de serviços de saúde não mostrou diferenças estatisticamente significativas quanto ao preenchimento adequado da caderneta da criança (Tabela 3 ).
$\mathrm{Na}$ análise ajustada, a escolaridade da mãe permaneceu associada ao desfecho. As crianças cujas mães tinham nove anos ou mais de estudo apresentaram uma chance quase seis vezes maior de terem a caderneta da criança preenchida adequadamente quando comparadas as crianças com mães com menor nível de escolaridade. As demais variáveis incluídas na análise perderam significância após o ajuste (Tabela 4).

Tabela 2

Razão de prevalência bruta da associação do preenchimento adequado das cadernetas de saúde e características biológicas das mães e das crianças. Caracol e Anísio de Abreu, Piauí, 2008.

\begin{tabular}{|c|c|c|c|c|c|c|}
\hline Variáveis & $\mathbf{N}$ & $\%$ & $\begin{array}{l}\text { Prevalência de } \\
\text { adequação (\%) }\end{array}$ & $\mathbf{R P}$ & $\mathrm{IC} 95 \%$ & $p$ \\
\hline Idade da mãe (anos) & & & & & & 0,77 * \\
\hline $20-29$ & 196 & 57,3 & 21,4 & 1,00 & & \\
\hline 30 ou mais & 40 & 11,7 & 25,0 & 1,17 & $0,64-2,13$ & \\
\hline Menos de 20 & 106 & 31,0 & 22,6 & 1,06 & $0,68-1,64$ & \\
\hline Cor da pele da mãe & & & & & & $0,79 *$ \\
\hline Preta & 7 & 2,0 & 14,3 & 1,00 & & \\
\hline Parda/mulata & 287 & 83,9 & 22,0 & 1,54 & $0,25-9,56$ & \\
\hline Branca & 48 & 14,0 & 25,0 & 1,75 & $0,27-11,46$ & \\
\hline Número de filhos & & & & & & $0,42 *$ \\
\hline 4 ou mais & 34 & 10,1 & 14,7 & 1,00 & & \\
\hline 3 & 37 & 11,0 & 21,6 & 1,47 & $0,53-4,06$ & \\
\hline 2 & 118 & 35,0 & 20,3 & 1,38 & $0,57-3,35$ & \\
\hline 1 & 148 & 43,9 & 26,4 & 1,79 & $0,76-4,21$ & \\
\hline Idade da criança & & & & & & 0,25 ** \\
\hline$\geq 1$ ano & 150 & 43,9 & 20,0 & 1,00 & & \\
\hline 6 meses $<1$ ano & 138 & 40,4 & 22,5 & 1,12 & $0,72-1,75$ & \\
\hline $0<6$ meses & 54 & 15,8 & 27,8 & 1,39 & $0,81-2,37$ & \\
\hline
\end{tabular}

* qui-quadrado $p$-valor de Pearson; ** qui-quadrado $p$-valor de linearidade. 
Tabela 3

Razão de prevalência bruta da associação do preenchimento adequado das cadernetas de saúde e utilização de serviços de saúde. Caracol e Anísio de Abreu, Piauí, 2008.

\begin{tabular}{|c|c|c|c|c|c|c|}
\hline Variáveis & $\mathbf{N}$ & $\%$ & $\begin{array}{l}\text { Prevalência de } \\
\text { adequação (\%) }\end{array}$ & $\mathbf{R P}$ & IC95\% & $p^{*}$ \\
\hline Mãe realizou pré-natal & & & & & & 0,68 \\
\hline Não & 7 & 2,0 & 28,6 & 1,00 & & \\
\hline Sim & 335 & 98,0 & 22,1 & 0,77 & $0,22-2,54$ & \\
\hline Pré-natal & & & & & & 0,14 \\
\hline Inadequado & 268 & 80,0 & 23,9 & 1,00 & & \\
\hline Adequado & 67 & 20,0 & 14,9 & 0,63 & $0,34-1,15$ & \\
\hline Consultou no puerpério & & & & & & 0,15 \\
\hline Não & 245 & 71,6 & 20,0 & 1,00 & & \\
\hline Sim & 97 & 28,4 & 27,8 & 1,39 & $0,93-2,09$ & \\
\hline Criança consultou nos & & & & & & 0,51 \\
\hline \multicolumn{7}{|l|}{ últimos 3 meses } \\
\hline Não & 143 & 41,8 & 20,3 & 1,00 & & \\
\hline Sim & 199 & 58,2 & 23,6 & 1,16 & $0,77-1,75$ & \\
\hline \multicolumn{7}{|l|}{ últimos 3 meses } \\
\hline Não & 333 & 97,4 & 21,9 & 1,00 & & \\
\hline Sim & 9 & 2,6 & 33,3 & 1,52 & $0,59-3,92$ & \\
\hline Hospitalização da criança nos & & & & & & 0,23 \\
\hline \multicolumn{7}{|l|}{ últimos 12 meses } \\
\hline $\operatorname{Sim}$ & 29 & 8,5 & 31,0 & 1,00 & & \\
\hline Não & 313 & 91,5 & 21,4 & 0,69 & $0,39-1,24$ & \\
\hline
\end{tabular}

* qui-quadrado $p$-valor de Pearson. 
Razão de prevalência ajustada da associação do preenchimento adequado das cadernetas de saúde. Caracol e Anísio de Abreu, Piauí, 2008

\begin{tabular}{|c|c|c|c|}
\hline Variáveis & Razão de Prevalência & IC95\% & $p$ \\
\hline Municípioa & & & 0,42 \\
\hline Caracol & 1,00 & & \\
\hline Anísio de Abreu & 1,17 & $0,79-1,74$ & \\
\hline Áreaa & & & 0,68 \\
\hline Rural & 1,00 & & \\
\hline Urbana & 1,09 & $0,72-1,66$ & \\
\hline Renda familiara(SM) & & & 0,56 \\
\hline Menos de 1 & 1,00 & & \\
\hline 1 a 1,9 & 1,03 & $0,63-1,69$ & \\
\hline 2 ou mais & 1,19 & $0,74-1,91$ & \\
\hline Escolaridade da mãea (anos) & & & $<0,001$ \\
\hline 0 a 4 & 1,00 & & \\
\hline 5 a 8 & 3,34 & $1,33-8,34$ & \\
\hline 9 ou mais & 5,67 & $2,24-14,36$ & \\
\hline Escolaridade do paia (anos) & & & 0,84 \\
\hline 0 a 4 & 1,00 & & \\
\hline 5 a 8 & 0,97 & $0,58-1,63$ & \\
\hline 9 ou mais & 0,98 & $0,59-1,62$ & \\
\hline Bolsa Famíliaa & & & 0,16 \\
\hline Sim & 1,00 & & \\
\hline Não & 1,44 & $0,87-2,4$ & \\
\hline Pré-natalb & & & 0,26 \\
\hline Inadequado & 1,00 & & \\
\hline Adequado & 0,39 & $-1,05-0,28$ & \\
\hline Consultou no puerpériob & & & 0,36 \\
\hline Não & 1,00 & & \\
\hline Sim & 0,22 & $-0,25-0,7$ & \\
\hline
\end{tabular}

a Nível hierárquico da variável no modelo teórico de análise; b Ajustada para escolaridade da mãe.

\section{Discussão}

O estudo mostrou que menos de um quarto das crianças tinha a caderneta de saúde com preenchimento adequado, ou seja, um precário registro de dados importantes, revelando pouca importância à puericultura e evidenciando a fragilidade da assistência para um procedimento de baixa complexidade, que não necessita participação de pessoal com qualificação de nível superior.

O desfecho do presente estudo foi elaborado levando-se em conta a presença de dados de identificação das crianças, atenção ao desenvolvimento e crescimento, bem como preenchimento do calendário vacinal, tidos como aspectos fundamentais da assistência à saúde das crianças. Considerouse a presença de dados de identificação das crianças como essencial, na medida em que possibilitava sua localização permitindo a procura e intervenção ativa naquelas faltosas ao Programa e nas mais vulneráveis. ${ }^{16} \mathrm{~A}$ incorporação do monitoramento do desenvolvimento foi embasada em estudos que comprovaram essa necessidade. $17,18 \mathrm{O}$ acompanhamento do crescimento foi representado pela presença de marcação do peso da criança na caderneta. ${ }^{19,20} \mathrm{O}$ último componente utilizado na construção do desfecho foi presença de dados de imunização, reconhecidamente a ação de saúde com maior impacto na prevenção de doenças. ${ }^{21}$

Partiu-se da presunção que a boa prática estava refletida em registros adequados e que procedimentos não realizados não foram preenchidos. 22

Evidentes limitações devem ser ressaltadas na construção do desfecho do presente estudo. Por exemplo, não se considerou a completude dos dados de identificação, não se levou em conta a atualização 
do acompanhamento do desenvolvimento ou do número de pesos anotados em relação à idade das crianças. Não se verificou também se a vacinação estava atualizada. Assim, o desfecho foi estabelecido na mera presença simultânea de registros nas respectivas cadernetas.

Investigações realizadas em diversas localidades do Brasil e que avaliaram o preenchimento das cadernetas de saúde também mostraram resultados negativos. Em relação aos componentes do desfecho da presente investigação, verificou-se expressiva ausência nos dados de identificação, 23 falhas no preenchimento das curvas de crescimento 5,24 e pequeno percentual de acompanhamento do desenvolvimento. ${ }^{24,25}$ Ao analisarem a qualidade do preenchimento de forma total, os estudos mostraram precária utilização da caderneta de saúde da criança25,26 ou a classificaram como "grosseiramente deficiente". 27

$\mathrm{Na}$ análise verificou-se que as crianças com mães de maior escolaridade tiveram maior prevalência de cadernetas adequadamente preenchidas. Escolaridade pode ser uma variável proxy de posição social e no caso tal achado denotaria as indesejáveis iniquidades no sistema de saúde local, uma vez crianças mais vulneráveis receberam piores cuidados. Por outro lado, a caderneta de saúde é um instrumento que permite aos pais o acompanhamento das condições de saúde da criança e mães de maior escolaridade poderiam exigir essas informações das equipes de saúde. Outros estudos mostraram que maior escolaridade materna estava associada à melhor preenchimento da caderneta.23,24

As variáveis de utilização de serviços de saúde não alcançaram significância estatística o que pode evidenciar deficiências nas ações de saúde. Se a atenção fosse continuada e integral, tanto as mães que realizaram pré-natal e, principalmente, aquelas classificadas como adequadas (início precoce e maior número de consultas) deveriam estar sensibilizadas para o atendimento dos seus filhos, especialmente durante o primeiro ano de vida. De forma semelhante, consultar durante o puerpério, que significava contato com serviços de saúde após o nascimento do filho também deveria apontar para cuidados redobrados de puericultura.

Ainda que as variáveis sobre procura de serviços ambulatoriais de saúde fossem restritas aos três últimos meses constatou-se que consultar também não implicou em melhor preenchimento do instrumento, nem mesmo quando o motivo da consulta foi exclusivamente "puericultura".

A análise mostrou que entre as 16 crianças com baixo peso ao nascimento, nenhuma apresentava caderneta preenchida de maneira adequada. Esse achado também expressou a má qualidade da assistência, uma vez que apresentar baixo peso ao nascimento, 1 reconhecido fator para mortalidade infantil, também não favoreceu as condições de acompanhamento.

Não se supôs que a inclusão das outras crianças com outros modelos de cadernetas, as quais contavam com menos informações, alterassem os resultados observados no presente estudo. Afinal a análise mostrou que quase $70 \%$ das crianças não tinham nenhum registro sobre acompanhamento do desenvolvimento, $30 \%$ não tinham o peso anotado e $8 \%$ não receberam nenhuma imunização, demonstrando as fragilidades da assistência. Os resultados apontam para a necessidade de capacitar, conscientizar e estimular os profissionais de saúde da rede pública quanto à importância da caderneta de saúde da criança, uma vez que trata-se de importante instrumento que permite o acompanhamento longitudinal e integral da saúde da criança.

\section{Referências}

1. Barros FC, Matijasevich A, Requejo JH, Giugliani E, Maranhão AG, Monteiro CA, Barros AJD, Bustreo F, Merialdi M, Victora CG. Recent trends in maternal, newborn, and child health in Brazil: progress toward Millennium development goals 4 and 5. Am J Public Health. 2010; 100 (10): 1877-89.

2. PNUD (Programa das Nações Unidas para o Desenvolvimento). Atlas do Desenvolvimento Humano. Índice de Desenvolvimento Humano - Municipal, 1991 e 2000 [serial online] [cited $2011 \mathrm{dez} 7$ ]. Disponível em http://www.pnud.org.br/atlas/ranking/IDH-M\%2091\%2000 $\% 20$ Ranking $\% 20$ decrescente $\% 20$ (pelos $\% 20$ dados $\%$ 20de\%202000).htm
3. Ministério da Saúde. Manual para utilização da caderneta de saúde da criança. Brasília, DF; 2005.

4. Victora CG, Aquino EML, Leal MC, Monteiro CA, Barros FC, Szwarcwald CL. Saúde de mães e crianças no Brasil: progressos e desafios. Lancet. 2011; 32-46.

5. Santos SR, Cunha AJLA, Gamba CM, Machado FG, Leal Filho JMM, Moreira NLM. Avaliação da assistência à saúde da mulher e da criança em localidade urbana na região Sudeste do Brasil. Rev Saúde Pública. 2000; 34 (3): 26671

6. Blank D. A puericultura hoje: um enfoque apoiado em evidências. J Pediatr (Rio J). 2003; 79 (Supl.1): S13-22. 
7. Brasil. Ministério da Saúde. Departamento Nacional de Auditoria do SUS: Saúde atualiza e amplia a caderneta de saúde da criança. Brasília, DF; 2007.

8. Faria M, Nogueira TA. Avaliação do uso da caderneta de saúde da criança nas unidades básicas de saúde em um município de Minas Gerais. Rev Bras Ciênc Saúde. 2013; 11: 8-15.

9. Linhares AO, Gigante DP, Bender E, Cesar JA. Avaliação dos registros e opinião das mães sobre a caderneta de saúde da criança em unidades básicas de saúde, Pelotas, RS. Rev AMRIGS. 2012; 56 (3): 245-50.

10. Cesar JA. Indicadores de Saúde Materno-Infantil no Semiárido: Lista A-Z para Prioridades de Intervenção na Assistência a Gestação e ao Parto e no Estado Nutricional. Relatorio de Pesquisa. Pastoral da Crianca/Unicef; 2011.

11. Cesar JA. Descrevendo o perfil, identificando fatores associados e promovendo intervenção: a gravidez na adolescência em municípios de pequeno porte no Nordeste do Brasil. Relatório Técnico. Brasília, DF: CNPq; 2012.

12. Brasil. Ministério da Saúde. Sistema de Informações sobre Mortalidade. Brasília, DF; 2009.

13. Brasil. Ministério da Saúde. Assistência pré-natal: manual puerpério. Brasília, DF; 2006.

14. Barros AJD, Hirakata UM. Alternatives for logistic regression in cross-sectional studies: an empirical comparision of models that directly estimate the prevalence ratio. BMC Med Res Methodol. 2003; 3: 21.

15. Victora CG, Huttly SR, Fuchs SC, Olinto MTA. The role of conceptual frameworks in epidemiological analysis: a hierarchical approach. Int J Epidemiol. 1997; 26 (1): 224-47.

16. Del Ciampo LA, Ricco RG, Daneluzzi JC, Del Ciampo IRL, Ferraz IS, Almeida CAN. O programa de saúde da família e a puericultura. Ciên Saúde Colet. 2006; 11 (3): 739-43.

17. American Academy of Pediatrics, Committee on Children with Disabilities. Developmental surveillance and screening of infants and young children. Pediatrics. 2001; 108: $192-6$.
18. Glascoe FP. Developmental, behavioral, and educational surveillance. In: Green FP, Haggerty RJ, Weitzman M, editors. Ambulatory pediatrics. Philadelphia: WB Saunders; 1999; p. 90-105.

19. Garner P, Panpanich R, Logan S. Is routine growth monitoring effective? A systematic review of trials. Arch Dis Child. 2000; 82: 197-201.

20. Romani SAM, Lira PIC. Fatores determinantes do crescimento infantil. Rev Bras Saúde Matern Infant. 2004; 4 (1): 15-23.

21. U.S. Preventive Services Task Force. Childhood immunizations. U.S.preventive services task force. Guide to clinical preventive services. Baltimore: Williams \& Wilkins; 1996, p.790.

22. Panacek EA. Performing Chart Review Studies. Air Med J. 2007; 26: 206-10.

23. Goulart LMHF, Alves CRL, Viana MRA, Moulin ZS, Carmo GAA, Costa JGD, Almeida JSCB. Caderneta de saúde da criança: avaliação do preenchimento dos dados sobre gravidez, parto e recém-nascido. Rev Paul Pediatr. 2008; 26 (2): 106-12.

24. Vieira GO, Vieira TO, Costa MCO, Netto PVS, Cabral VA. Uso do cartão da criança em Feira de Santana, Bahia. Rev Bras Saúde Matern Infant. 2005; 5 (2): 177-84.

25. Ceia MLM, Cesar JA. Avaliação do preenchimento dos registros de puericultura em unidades básicas de saúde em Pelotas, RS. Revista AMRIGS. 2011; 55 (3): 244-9.

26. Alves CRL, Lasmar LMLDF, Goulart LMHF, Alvim CG, Maciel GVR, Viana MRA, Colosimo EA, Carmo GAA, Costa JGD, Magalhães MEN, Mendonça ML, Beirão MMV, Moulin ZS. Qualidade do preenchimento da caderneta de saúde da criança e fatores associados. Cad Saúde Pública. 2009; 25 (3): 583-95.

27. Ratis CAS, Batista Filho M. Aspectos estruturais e processuais da vigilância do crescimento de menores de cinco anos em serviços públicos do Estado de Pernambuco. Rev Bras Epidemiol. 2004; 7 (1): 44-53.

Recebido em 30 de outubro de 2013

Versão final apresentada em 20 de maio de 2014

Aprovado em 26 de junho de 2014 